\title{
Conformal bootstrap signatures of the tricritical Ising universality class
}

\author{
Chethan N. Gowdigere®, Jagannath Santara, and Sumedha@ \\ School of Physical Sciences, National Institute of Science Education and Research, Jatni 752050, India \\ and Homi Bhabha National Institute, Training School Complex, Anushakti Nagar, Mumbai 400094, India
}

(Received 28 November 2018; revised manuscript received 17 April 2020; accepted 12 June 2020; published 30 June 2020)

\begin{abstract}
We study the tricritical Ising universality class using conformal bootstrap techniques. By studying bootstrap constraints originating from multiple correlators on the conformal field theory (CFT) data of multiple operator product expansions (OPEs), we are able to determine the scaling dimension of the spin field $\Delta_{\sigma}$ in various noninteger dimensions $2 \leq d \leq 3$. Here, $\Delta_{\sigma}$ is connected to the critical exponent $\eta$ that governs the (tri)critical behavior of the two-point function via the relation $\eta=2-d+2 \Delta_{\sigma}$. Our results for $\Delta_{\sigma}$ match with the exactly known values in two and three dimensions and are a conjecture for noninteger dimensions. We also compare our CFT results for $\Delta_{\sigma}$ with $\epsilon$-expansion results, available up to $\epsilon^{3}$ order. Our techniques can be naturally extended to study higher-order multicritical points.
\end{abstract}

DOI: 10.1103/PhysRevD.101.116020

\section{INTRODUCTION}

The consequences of the conformal hypothesis which posits conformal invariance to the behavior of physical systems at criticality, in addition to scale invariance, are most far reaching in two dimensions, where the conformal symmetry is the infinite-dimensional Virasoro algebra. The seminal work of Belavin, Polyakov, and Zamoldchikov $[1,2]$ resulted in the discovery of a whole class of twodimensional conformal field theories (CFTs) viz. the Virasaro minimal models. Each of these models describes a universality class, and the exact knowledge of the scaling dimensions of the operators amounts to a derivation of the critical exponents purely from conformal invariance. The Ising model, the tricritical Ising model, and the threeand four-state Potts models in two dimensions were thus exactly solved 30 years ago. The infinitude of conformal symmetry made two dimensions rather special, and the use of conformal field theories to study critical phenomena was restricted to two dimensions alone.

Recent times have seen a breakthrough in doing the same for three and other dimensions. Even though the conformal group is finite dimensional now, technical advances made in the explicit computation of (global) conformal blocks (first in [3-5] and later in [6-8]) resulted in astounding progress (see [9] for a recent review) and has provided the most precise values [10-13] for the critical exponents of the three-dimensional Ising model. Rychkov et al. analyzed

Published by the American Physical Society under the terms of the Creative Commons Attribution 4.0 International license. Further distribution of this work must maintain attribution to the author(s) and the published article's title, journal citation, and DOI. Funded by SCOAP. the restrictions imposed by conformal invariance and found that the conformal field theory corresponding to the Ising universality class sits on the boundary of the allowed region at a kinklike point in the space of scaling dimensions of the only two relevant operators. Furthermore, remarkably, they could extend this analysis to all noninteger dimensions between two and four, and they showed that even here, the theory corresponding to the Ising universality class is always located at a kinklike point [14]. Hitherto [15-20], only critical points have been studied using conformal field theory methods. Other critical points (tricritical and multicritical) also have conformal symmetry, and here we use conformal field theory techniques to study tricritical points. In this paper, we look at the tricritical Ising point in two and higher dimensions and show that just like the Ising critical point, the tricritical Ising point can also be recognized by its special signatures in the space of scaling dimensions of the appropriate operators.

A tricritical point is a fixed point where three critical lines and a line of first-order transitions meet (or a point where three coexisting phases simultaneously become critical) [21]. Quantum scalar field theory with a $\phi^{6}$ interaction provides one realization of this universality class. Here, there is a line of Ising critical points which end in a higher-order critical point, namely, the tricritical point which has a different set of critical exponents. These two critical behaviors have different upper critical dimensions: three for the tricritical Ising point and four for the Ising critical point [22]. Thus, field theory and renormalization group based techniques such as the $\epsilon$-expansion have had limited success $[23,24]$. CFT techniques avoid the flow and only study the fixed point, which is where the extra scaling and conformal symmetries are present. Yet, so far, a CFT 
study of the tricritical point has not had as much success as the critical point, in dimensions other than two.

The paper is organized as follows. We start with a brief review of CFT in Sec. II. We recall the prime principles of CFT, the essential details of the conformal bootstrap program, and some key results obtained so far in the bootstrap program that we need for our analysis of the tricritical CFT. In Sec. III, we present the bootstrap analysis that allows us to compute the tricritical exponent $\eta$ for all noninteger dimensions between two and three. In the same section, we compare our results with the best available $\epsilon$-expansion results for $\eta$. In Sec. IV, we present a further bootstrap analysis pertaining to multiple universality classes and multicriticality. We conclude the paper in Sec. V with a summary of results and future directions.

\section{BRIEF REVIEW OF CFT}

We first provide a quick overview of the prime principles of CFT: CFT data, the operator product expansion (OPE), unitarity constraints, and crossing symmetry constraints. A CFT is specified (partially) by a list of local primary operators a.k.a. scaling operators (each operator is a primary under the global conformal symmetry and is specified by its scaling dimension and spin). The scaling dimension of a local operator in a unitary CFT is bounded from below depending on its spin. A product of two such local operators is expandable in terms of all the local operators [25,26]; this is known as the operator product expansion,

$$
\phi_{1}\left(x_{1}\right) \phi_{2}\left(x_{2}\right)=\sum_{\mathcal{O}} \lambda_{12 \mathcal{O}} C\left(x_{12}, \partial_{x_{2}}\right) \mathcal{O}\left(x_{2}\right)
$$

where $C\left(x_{12}, \partial_{x_{2}}\right)$ is fully determined by conformal invariance and $\lambda_{12 \mathcal{O}}$ is referred to as an OPE coefficient, which is real in a unitary CFT. The set of local primary operators and the set of OPE coefficients are together referred to as CFT data, which completely specifies a local CFT.

Critical exponents are encoded in the scaling dimensions of only a few low-lying primary operators (the relevant ones). There are only two relevant operators in the Ising CFT, while there are four relevant operators in the CFT of the tricritical Ising point [27]. Of the four, one only needs three to define the critical exponents of the tricritical Ising universality class. In two dimensions these exponents are known exactly [1,2]; the CFT is the second Virasaro minimal model consisting of six primary operators (while the Ising CFT is the first Virasaro minimal model consisting of three primary operators).

In a CFT, a four-point function, shown here for scalar operators, is mostly fixed by conformal invariance, except for an arbitrary function of the two independent cross ratios $u=\frac{x_{12}^{2} x_{34}^{2}}{x_{13}^{2} x_{24}^{2}}$ and $v=\frac{x_{14}^{2} x_{23}^{2}}{x_{13}^{2} x_{24}^{2}}$ with $x_{12} \equiv\left|\vec{x}_{1}-\vec{x}_{2}\right| \ldots$ :

$$
\left\langle\phi\left(x_{1}\right) \phi\left(x_{2}\right) \phi\left(x_{3}\right) \phi\left(x_{4}\right)\right\rangle \sim G(u, v) .
$$

Using OPEs one can evaluate the four-point function in terms of the CFT data

$$
G(u, v)=\sum_{\mathcal{O}} \lambda_{12 \mathcal{O}} \lambda_{34 \mathcal{O}} G_{\mathcal{O}}(u, v),
$$

where the summation is over the primary operators that occur in both the $\phi_{1} \times \phi_{2}$ and the $\phi_{3} \times \phi_{4}$ OPEs and $G_{\mathcal{O}}(u, v)$ is the (global) conformal block. This evaluation of the four-point function can be done in two different ways, and both ways agree that

$$
\sum_{\mathcal{O}} \lambda_{12 \mathcal{O}} \lambda_{34 \mathcal{O}} G_{\mathcal{O}}(u, v)=\sum_{\mathcal{O}^{\prime}} \lambda_{14 \mathcal{O}^{\prime}} \lambda_{23 \mathcal{O}^{\prime}} G_{\mathcal{O}^{\prime}}(u, v)
$$

puts a constraint on the CFT data, which is referred to as the bootstrap equation or the crossing symmetry constraint. The conformal bootstrap program employs Eq. (4) (every four-point function provides one equation) to search for CFTs by successively constraining the CFT data. This equation was shown to be tractable numerically starting with the seminal work [10] and leading up to [28-32], the main tools for the results of this paper.

The first important step in the numerical conformal bootstrap program [10] is to focus on a certain region of the two-dimensional space of conformal cross ratios, now known as the spacelike diamond, in which the conformal blocks $G_{\mathcal{O}}(u, v)$ turn out to be real and analytic and hence have Taylor expansions. Equation (4) is a vector equation in a real analytic function space. This function space is coordinatized as follows [10], and this is inspired by the knowledge of how Eq. (4) is solved in the free scalar CFT. There is a certain point where the convergence of the infinite sums in (4) is fastest (for the free scalar CFT): $z=\frac{1}{2}, \bar{z}=\frac{1}{2}$, the crossing-symmetric point. The $z, \bar{z}$ coordinates for the space of conformal cross ratios are related to the original coordinates $u, v$ by $u=z \bar{z}, v=(1-z)(1-\bar{z})$. Every element of the real analytic function space in which the conformal blocks live is parametrized by the coefficients in its Taylor expansion around this $z=\frac{1}{2}, \bar{z}=\frac{1}{2}$ point, an infinite-tuple of real numbers. Thus, the values of a real analytic function and the values of its various mixed partial derivatives at this special point form the coordinates for this function space. Now, one solves Eq. (4) by truncating to a finite number of coordinates, depending on the accuracy needed. The truncation is specified by the maximum number of derivatives retained (with respect to both $z$ and $\bar{z}$ ). In obtaining the results of this paper, we have used the semidefinite program solver [29] via the PyCFTBoot wrapper [30] to implement the numerical conformal bootstrap. Other resources to implement the numerical bootstrap can be found in $[28,31,32]$. 
For the last part of the CFT review, we recall two results that we will need, which have already been obtained via the numerical conformal bootstrap. Both originate from the crossing symmetry constraints on the four-point function $\langle\phi \phi \phi \phi\rangle$ of a single scalar operator.

(i) The first result is the bound obtained by Rattazzi, Rychkov, Tonni, and Vichi in [10], which we refer to as the "RRTV bound." In [10], it was shown that the bootstrap equation requires that the operator in the $\phi \times \phi$ OPE with the lowest scaling dimension is always a scalar, referred to as the lowest scalar, and its scaling dimension is bounded from above. This bound is a function of the scaling dimension of $\phi$ $\left(\Delta_{\phi}\right)$ and is determined numerically in all dimensions.

(ii) The second result is what we refer to as the "Rychkov bound" [11]. This is a bound on the scalar operator in the $\phi \times \phi$ OPE with the next-tolowest scaling dimension, if present, which, unlike the operator with the lowest scaling dimension, need not be a scalar. The result is that there is an upper bound, determined numerically, for any set of values for $\Delta_{\phi}$ and for the scaling dimension of the lowest scalar (which is already constrained by the RRTV bound).

\section{SCALING DIMENSION OF THE LOWEST SCALAR}

In this paper, we study a class of CFTs whose low-lying operator spectrum includes four relevant scalars $\sigma, \epsilon, \sigma^{\prime}, \epsilon^{\prime}$ in the increasing order of scaling dimensions. The twodimensional tricritical Ising model [1,2] is one example; there, the $\sigma$ 's are the $\mathbf{Z}_{2}$-odd operators, while the $\epsilon$ 's are the $\mathbf{Z}_{2}$-even operators. In the $\phi^{6}$ realization, the four relevant scalars are $\sigma \leftrightarrow \phi, \epsilon \leftrightarrow \phi^{2}, \sigma^{\prime} \leftrightarrow \phi^{3}, \epsilon^{\prime} \leftrightarrow \phi^{4}$. There are ten OPEs that concern these four fields, but we focus only on two of them viz. the $\sigma \times \sigma$ and $\epsilon \times \epsilon$ OPEs since the four-point functions whose bootstrap constraints we focus on in this paper, viz. $\langle\sigma \sigma \sigma \sigma\rangle$ and $\langle\epsilon \epsilon \epsilon \epsilon\rangle$, only need these two OPEs for their evaluation. Furthermore, the class of CFTs we study are those with the following particular OPEs:

$\sigma \times \sigma=\mathbf{1}+\epsilon+\epsilon^{\prime}+\ldots, \quad \epsilon \times \epsilon=\mathbf{1}+\epsilon^{\prime}+\ldots$

That is, the global conformal families of $\epsilon$ and $\epsilon^{\prime}$ are present in the $\sigma \times \sigma \mathrm{OPE}$, and the global conformal family of $\epsilon^{\prime}$ is present in the $\epsilon \times \epsilon \mathrm{OPE}$. Note that $\epsilon$ is the scalar operator with the lowest scaling dimension that is present in the $\sigma \times \sigma \mathrm{OPE}$, and it is referred to as the "lowest scalar" of the OPE; similarly, $\epsilon^{\prime}$ is the "next-to-lowest scalar" of the OPE. Also note that $\epsilon^{\prime}$ is the lowest scalar of the $\epsilon \times \epsilon$ OPE. Thus, $\epsilon^{\prime}$ is the lowest scalar in one OPE and the next-to-lowest scalar in another. This is the problem we study here using the numerical conformal bootstrap-CFTs (in all dimensions, two to three and beyond) with a low-lying spectrum and OPEs given by (5) [33] — and we find that the bootstrap constraints reflect many aspects of tricritical phenomena.

In this paper, we work only with unitary CFTs and use the unitary conformal bootstrap for our analysis. Although it is known that CFTs in fractional dimensions are nonunitary, a fact which was first encountered in [36] and further expounded in [37] (see also [38]). As was shown in [36], nonunitarity manifests itself in the form of the existence of negative norm states at high dimensions. To quote that work, "... a few negative norm states at high dimensions, hidden among lots of positive-norm states of comparable dimensions, probably do not have a strong effect on the low-energy physics. In a recent conformal bootstrap study of the Wilson-Fisher fixed point in fractional dimensions [14], it was assumed that these theories were unitary, and very reasonable results were obtained ..." In the present paper, since we are studying low-dimensional operators (the relevant operators), much like [14], we expect that nonunitarity can be ignored and hence proceed with the unitary bootstrap analysis.

\section{A. Study in $d=2$}

We first present an analysis of the crossing symmetry constraints in two dimensions. We derive lessons from two dimensions and apply them to other dimensions in the next subsection. Our analysis below gives upper bounds on the scaling dimension of the operator $\epsilon^{\prime}$ for the class of CFTs under study.

\section{The first upper bound}

We obtain an upper bound on the scaling dimension of $\epsilon^{\prime}$ as follows. For a given value of $\Delta_{\epsilon}$, say $s$, we first find the RRTV bound on the lowest scalar in the $\epsilon \times \epsilon$ OPE which is a bound on $\Delta_{\epsilon^{\prime}}, r_{1}(s)$. We plot the points $\left(s, r_{1}(s)\right)$ in Fig. 1, where the $x$ axis is $\Delta_{\epsilon}$ and the $y$ axis is $\Delta_{\epsilon^{\prime}}$. We refer to this graph as the RRTV bound on $\Delta_{\epsilon^{\prime}}$. It is shown in Fig. 1 for two dimensions.

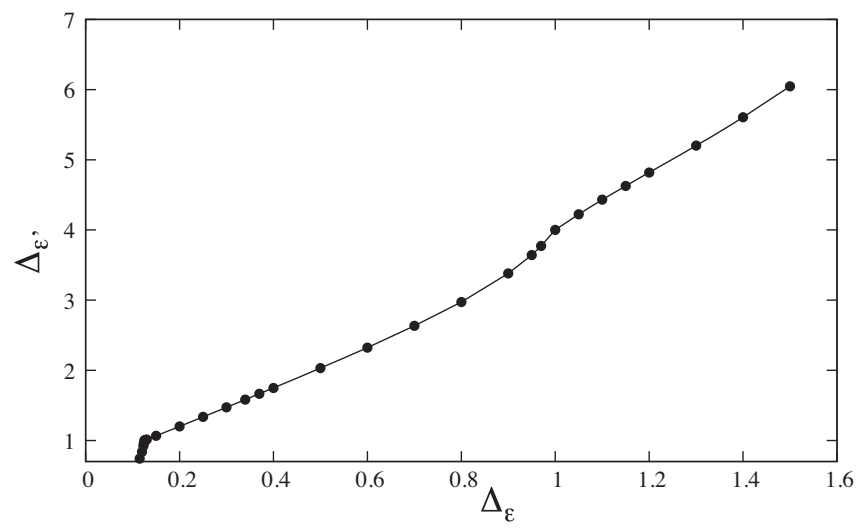

FIG. 1. The first upper bound on the scaling dimension of $\epsilon^{\prime}$ : the RRTV bound. 


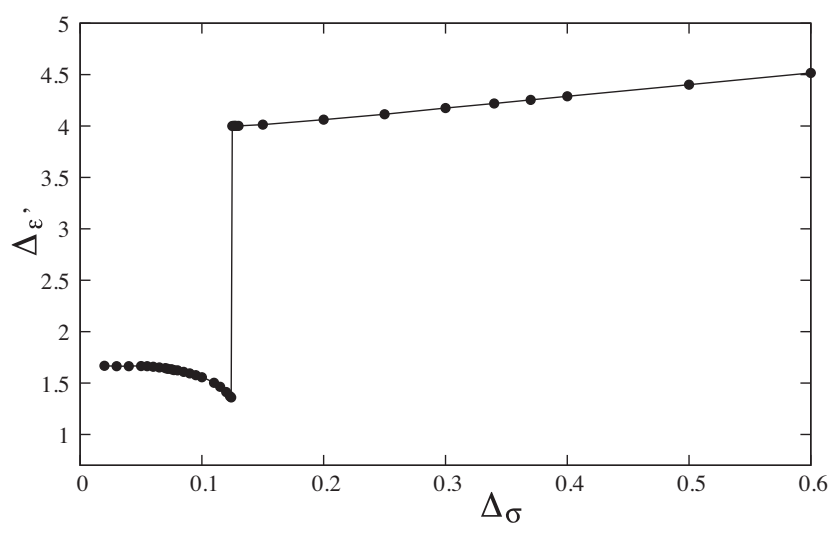

FIG. 2. The second upper bound on the scaling dimension of $\epsilon^{\prime}$ : the Rychkov bound.

\section{The second upper bound}

We obtain another upper bound on the scaling dimension of $\epsilon^{\prime}$ in the following way. For a given value of $\Delta_{\sigma}$, say $r$, we first find the RRTV bound on the lowest scalar in the $\sigma \times \sigma$ OPE, which is a bound on $\Delta_{\epsilon}, r_{2}(r)$. Then, for the pair $\left(\Delta_{\sigma}=r, \Delta_{\epsilon}=r_{2}(r)\right)$, we find the Rychkov bound on the next-to-lowest scalar in the $\sigma \times \sigma$ OPE which is a bound on $\Delta_{\epsilon^{\prime}}, r_{3}(r)$. We plot the points $\left(r, r_{3}(r)\right)$ in Fig. 2, where the $x$ axis is $\Delta_{\sigma}$ and the $y$ axis is $\Delta_{\epsilon^{\prime}}$. We refer to this graph as the Rychkov bound on $\Delta_{\epsilon^{\prime}}$. It is shown in Fig. 2 for two dimensions.

Now, we compare the RRTV and Rychkov bounds on $\Delta_{\epsilon^{\prime}}$. First, we note that the RRTV bound in Fig. 1 is a $\Delta_{\epsilon}-\Delta_{\epsilon^{\prime}}$ plot, while the Rychkov bound in Fig. 2 is a $\Delta_{\sigma}-\Delta_{\epsilon^{\prime}}$ plot. We can also convert the latter into a $\Delta_{\epsilon}-\Delta_{\epsilon^{\prime}}$ plot by plotting the points $\left(r_{2}(r), r_{3}(r)\right)$ instead of $\left(r, r_{3}(r)\right)$. We can now put the RRTV and Rychkov bounds in the same $\Delta_{\epsilon}-\Delta_{\epsilon^{\prime}}$ plot by plotting the points $\left(s, r_{1}(s)\right)$ and $\left(r_{2}(r), r_{3}(r)\right)$, respectively. This is shown for two dimensions in Fig. 3.

From Fig. 3 we observe that for smaller values of $\Delta_{\sigma}$ $(=r)$ [which corresponds to smaller values of $r_{2}(r)$ because $r_{2}(r)$ is a monotonically increasing function], we find that the Rychkov bound is bigger than the RRTV bound. For subsequent larger values of $\Delta_{\sigma}$, the graph for the Rychkov bound crosses the graph for the RRTV bound so that, to the left of the crossing point, the Rychkov bound is larger than the RRTV bound and, to the right of the crossing point, the RRTV bound is larger. There is a specific value for $\Delta_{\sigma}$, say $\Delta_{\sigma}^{\text {cross }}$, for which this crossing happens; to be precise, $r_{2}\left(\Delta_{\sigma}^{\text {cross }}\right)$ is the value on the $x$ axis for the crossing point. In two dimensions, the graphs for the RRTV and Rychkov bounds are plotted in Fig. 3, and we find $\Delta_{\sigma}^{\text {cross }}=0.075 \pm 0.001$. This compares well with the known value for $\Delta_{\sigma}$ in the two-dimensional tricritical Ising CFT (which is 0.075) within error bars. Hence, we conclude that the value of $\Delta_{\sigma}^{\text {cross }}$, determined by conformal bootstrap constraints as described above, is the value of $\Delta_{\sigma}$ in the CFT.

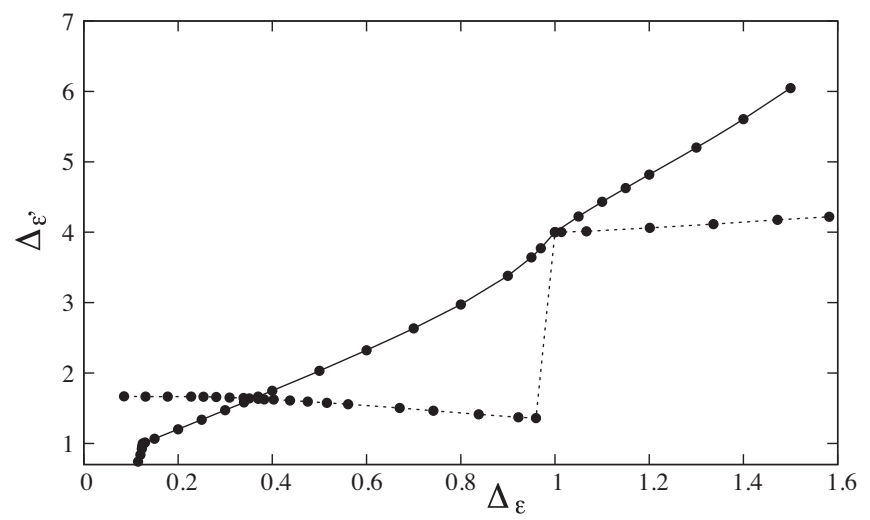

FIG. 3. The RRTV (solid line) and the Rychkov (dashed line) bounds in two dimensions. The two bounds cross at $r_{2}(0.075 \pm 0.001)$.

A more succinct description of the analysis of this subsection can be made as follows [39]: $\Delta_{\epsilon^{\prime}}$ can be maximized by keeping $\Delta_{\epsilon}$ fixed from the $\langle\epsilon \epsilon \epsilon \epsilon\rangle$ four-point function. Alternatively, $\Delta_{\epsilon^{\prime}}$ can be maximized in the $\langle\sigma \sigma \sigma \sigma\rangle$ four-point function, also keeping $\Delta_{\epsilon}$ to the maximal allowed value. Then, we observe that the tricritical Ising CFT in two dimensions satisfies these two maximization conditions simultaneously.

\section{Some details pertaining to the numerical study}

We used the program PyCFTBoot [30], which is a program written in PYTHON based on the SDPB solver [29]; these resources give us access to the semidefinite programming methods for the bootstrap pioneered in $[7,8,40]$. In all the computations and plots in this paper, we employed the following user-defined input parameters in PyCFTBoot: $k_{\max }=30, l_{\max }=30, m_{\max }=7, n_{\max }=10$. This gives us a table associated with conformal blocks (ConformalBlockTable in PyCFTBoot) with 30 poles, 30 spins, and a $7 \times 10$ triangle of derivatives (which amounts to working with $m_{\max }+2 n_{\max }=27$ derivatives of the conformal blocks). We computed with up to 40 poles, 40 spins, and an $8 \times 12$ triangle of derivatives (32 derivatives). This increased the computing time substantially without adding any substantial improvement in the details beyond what we report here. In the SDPB command used to bisect over gaps in a scalar operator sdp.bisect(lower, upper, tol, 0), we have changed from the default tolerance value of tol $=$ 0.01 to a tolerance value of tol $=0.00001$. This was essential to improve the precision of the Rychkov bound.

\section{B. $2<d<3$ and beyond}

The equations that encode the bootstrap constraints and, in fact, the whole formalism are analytic in the number of dimensions $d$ and thus provide a remarkable way to study CFTs in noninteger dimensions. The Ising model CFT was studied in all dimensions $2 \leq d \leq 4$ in [14]. Here, we study 


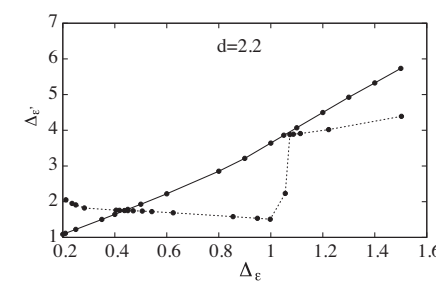

(a)

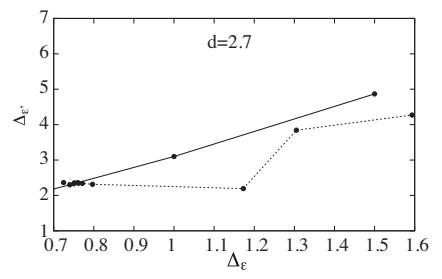

(c)

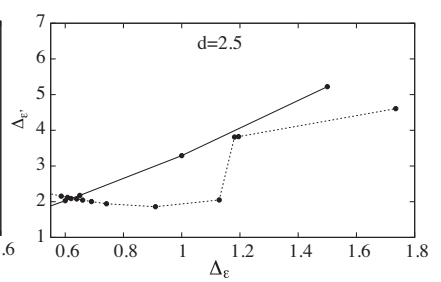

(b)

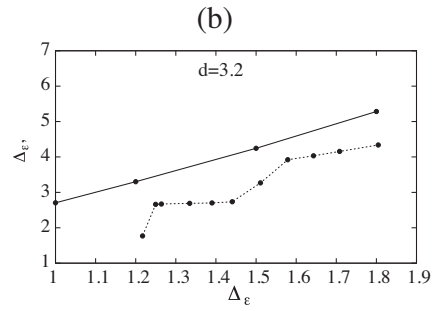

(d)

FIG. 4. The RRTV (solid line) and the Rychkov (dashed line) bounds in various noninteger dimensions: (a) $d=2.2$, (b) $d=2.5$, (c) $d=2.7$, and (d) $d=3.2$. The two bounds cross at (a) $r_{2}(0.136)$, (b) $r_{2}(0.259)$, (c) $r_{2}(0.352)$, and for (d) there is no crossing.

our class of tricritical Ising CFTs in a generic noninteger dimension $d$. We numerically determine the RRTV and Rychkov bounds and look for the point, if any, where the bounds coincide; from that point, we obtain $\Delta_{\sigma}^{\text {cross }}$ in the noninteger dimension $d$. The results of this investigation are presented in three ways. First, in Fig. 4, for four different representative noninteger dimensions $d=2.2$, 2.5, 2.7, 3.2, we plot the RRTV and Rychkov bounds and the point of intersection which gives $\Delta_{\sigma}^{\text {cross }}$. Second, in the first two columns of Table I, we give the $\Delta_{\sigma}^{\text {cross }}$ for more values of $d$ in the range $2<d<3$, where there is a crossing or intersection of the two bounds. Third, in Fig. 5, we show a comparison between the CFT computations $\Delta_{\sigma}^{\text {cross }}$ with the unitarity bound for scalar operators in the dimension range $2<d<3$.

First, we discuss the range of dimensions $2<d<3$ where there is a crossing of bounds. We find that $\Delta_{\sigma}^{\text {cross }}$ increases as $d$ increases from 2 to 3 (see Table I as well as Fig. 5). Note that $\Delta_{\sigma}^{\text {cross }}$ is bigger than the unitarity bound, $\frac{d-2}{2}$, for dimensions between 2 and 3 , and this difference is maximum for $d=2$ and decreases with increasing $d$. We also find that $\Delta_{\sigma}^{\text {cross }}$ approaches $\frac{1}{2}$ as $d$ approaches 3 . This matches with the known value for $\Delta_{\sigma}$ in $d=3$ : The upper critical dimension for the tricritical Ising model is 3 , wherein the scaling dimension of $\sigma$ is the classical value of $\frac{1}{2}$. The numerical computation becomes harder as $d \rightarrow 3$ because $\Delta_{\sigma}^{\text {cross }}$ seems to be very close to the unitarity bound.

Our numerical studies for $3<d<4$ show that the two bounds do not cross. See the fourth plot in Fig. 4. This observation is consistent with known facts. The tricritical Ising exponents for $3<d<4$ should be the same as for three dimensions, which is a violation of the unitarity bound (for $\Delta_{\sigma}$ ) and hence is in the realm of nonunitary

TABLE I. $\quad \Delta_{\sigma}$ in dimensions 2 to 3 from CFT analysis and from $\epsilon$-expansion.

\begin{tabular}{lcc}
\hline \hline Dimension $(\mathrm{d})$ & $\Delta_{\sigma}^{\text {cross }}$ from CFT & $\Delta_{\sigma}$ from $\epsilon$-expansion \\
\hline 3.00 & $\ldots$ & 0.50000 \\
2.90 & $\ldots$ & 0.45002 \\
2.80 & $0.4002(1)$ & 0.40014 \\
2.70 & $0.352(1)$ & 0.35043 \\
2.60 & $0.306(1)$ & 0.30098 \\
2.50 & $0.259(1)$ & 0.25184 \\
2.40 & $0.214(1)$ & 0.20311 \\
2.30 & $0.172(1)$ & 0.15486 \\
2.20 & $0.136(1)$ & 0.10716 \\
2.10 & $0.102(1)$ & 0.06010 \\
2.00 & $0.075(1)$ & 0.01374 \\
\hline \hline
\end{tabular}

CFTs. But our analysis, following [10,11], is an analysis of constraints on unitary CFTs.

Thus, our surmise that $\Delta_{\sigma}^{\text {cross }}$ gives the value for $\Delta_{\sigma}$ in the CFT has passed nontrivial tests by reproducing the known values in $d=2, d=3$ and in $3<d<4$, and hence the computations for $2<d<3$ constitute a prediction coming from conformal bootstrap analysis.

\section{Comparison with $\epsilon$-expansion}

Before the advent of bootstrap methods, $\epsilon$-expansion techniques were used to study CFTs in noninteger dimensions. The CFTs associated with the critical Ising model have been studied extensively in this way, starting with the seminal works of Wilson and Fisher [41,42]. For the Ising model ( $\phi^{4}$ theory), $\epsilon$-expansion results up to a very high order $\left(\epsilon^{5}\right)$ are available [43-45] (see also [46,47]). But the $\epsilon$-expansion results for tricritical CFTs ( $\phi^{6}$ theory) are not available to that high an order; computations up to $\epsilon^{3}$-order

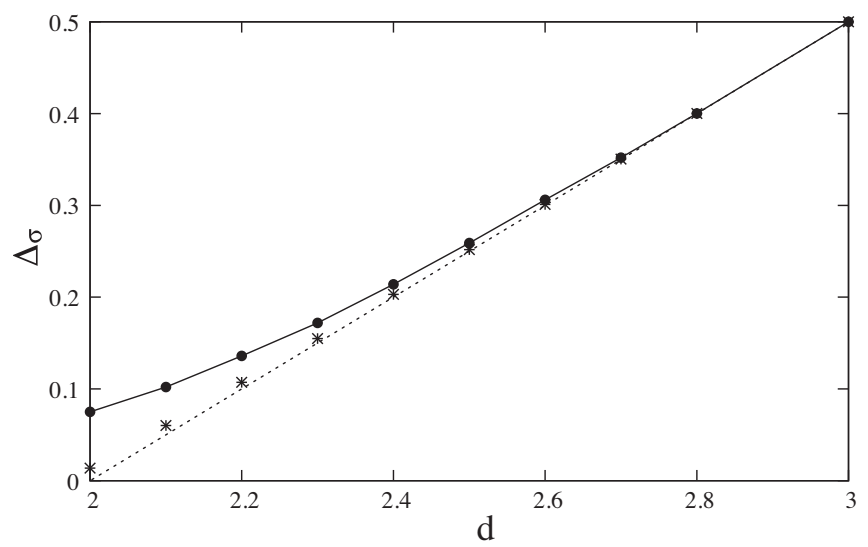

FIG. 5. The bold line shows the CFT computation for $\Delta_{\sigma}$, i.e., $\Delta_{\sigma}^{\text {cross }}$, and the dashed line shows the unitarity bound for scalar operators, for $2 \leq d \leq 3$. As the spatial dimension increases, $\Delta_{\sigma}^{\text {cross }}$ becomes very close to the unitarity bound. Points represented by $*$ that do not form a line represent the $\epsilon$-expansion computation for $\Delta_{\sigma}$. 
were done in $[23,24]$. From [48], we obtain the $\epsilon$-expansion up to order three for the critical exponent $\eta$; furthermore, using the relation between the critical exponent $\eta$ and the scaling dimension of the lowest scalar viz.

$$
\eta=2-d+2 \Delta_{\sigma}
$$

we have the following $\epsilon$-expansion result $(\epsilon=3-d)$ :

$$
\Delta_{\sigma}=\frac{1}{2}-\frac{\epsilon}{2}+\frac{\epsilon^{2}}{1000}+\frac{10125 \pi^{2}+91160}{15000000} \epsilon^{3}+\ldots
$$

We now test our hypothesis by comparing the values of $\Delta_{\sigma}^{\text {cross }}$ obtained above with the best-known values from $\epsilon$-expansion [Eq. (7)]. The comparison between the CFT results and $\epsilon$-expansion results is shown in Table I. It is also shown in Fig. 5 where the CFT values form a line and the $\epsilon$-expansion values are points $*$ that do not form a line. The results coincide in dimensions close to three but deviate as one approaches two dimensions. This is perhaps because the study of the tricritical Ising point using $\epsilon$-expansion is known to give poor estimates in two dimensions. One expects that modern approaches to $\epsilon$-expansion computations that incorporate conformal symmetry, such as the Rychkov-Tan [49] method and the Polyakov-Mellin bootstrap [20], would give better results [50].

\section{COEXISTENCE OF MULTIPLE UNIVERSALITY CLASSES AND MULTICRITICALITY}

\section{A. Two plateaus}

In the previous section, we started our discussion with a bootstrap analysis in two dimensions. Together with exact results available in two dimensions, we gathered information from the two-dimensional analysis and then applied it to dimensions other than two. In this section, we again focus on another observation about two dimensions. In the plot of the two-dimensional Rychkov bound (dashed line of Fig. 3), we see that there are two plateaus. One is the plateau that starts around $\Delta_{\epsilon}=1, \Delta_{\epsilon^{\prime}}=4$. This one has been well studied in [11]; it was argued that within this $\Delta_{\epsilon}-\Delta_{\epsilon^{\prime}}$ space, as one approaches the Ising CFT, the operator $\epsilon^{\prime}$ becomes irrelevant (in fact, it was used as one of the criteria to partially isolate the Ising CFT). Hence, this plateau can be associated with the Ising universality class.

Throughout the second plateau in Fig. $3, \Delta_{\epsilon}<1$ and $\Delta_{\epsilon^{\prime}}<2$. If a certain CFT is such that $\epsilon^{\prime}$ cannot be an irrelevant operator in it (such as the two-dimensional tricritical Ising CFT), then that CFT has to exist in the region $\left(\Delta_{\epsilon}<1\right)$ of this second plateau. We can thus associate this second plateau with the tricritical Ising universality class.

The two plateaus in the plot of the Rychkov bound indicate that the conformal bootstrap constraints allow for the (possible) existence of two different universality classes: one where $\epsilon^{\prime}$ is relevant and another where $\epsilon^{\prime}$ is irrelevant; in other words, the Ising and the tricritical Ising universality classes. This result from CFT analysis is consistent with our understanding from studies of $\phi^{6}$ field theory [22]: The $\epsilon^{\prime}$ operator is the $\phi^{4}$ operator, and it is known that the $\phi^{4}$ operator determines whether the flow is towards the tricritical point or the Ising point.

Thus, motivated by the above observations on plateaus in two dimensions, we plot the Rychkov bound in various noninteger dimensions in Fig. 6 and find that the twoplateau structure exists there, too. This is the conformal bootstrap signature for the existence of both the Ising and tricritical Ising universality classes in noninteger dimensions as well. On closer examination, we find that the width of the lower plateau decreases with increasing dimension and vanishes at $d=4$.

\section{B. The $\epsilon^{\prime \prime}$ operator}

So far, we have been working with the class of CFTs consisting of $\sigma, \epsilon, \epsilon^{\prime}$ operators in the lowest part of the spectrum with OPEs (5). Here, we introduce the operator above these, which is denoted by $\epsilon^{\prime \prime}$, and the OPEs are now

$\sigma \times \sigma=\mathbf{1}+\epsilon+\epsilon^{\prime}+\epsilon^{\prime \prime}+\ldots, \quad \epsilon \times \epsilon=\mathbf{1}+\epsilon^{\prime}+\ldots$

The two-dimensional tricritical Ising CFT is an example of this class of CFTs (from which the notation is derived) where $\epsilon^{\prime \prime}$ is the primary operator with the largest scaling dimension. In the $\phi^{6}$ realization, $\epsilon^{\prime \prime} \leftrightarrow \phi^{6}$.

We now analyze the bootstrap constraints for this class of CFTs. In two dimensions, when we fix $\Delta_{\sigma}$ and $\Delta_{\epsilon}$ to their exact values of 0.075 and 0.2 , respectively, the bootstrap equations from the $\langle\sigma \sigma \sigma \sigma\rangle$ correlator give an upper bound

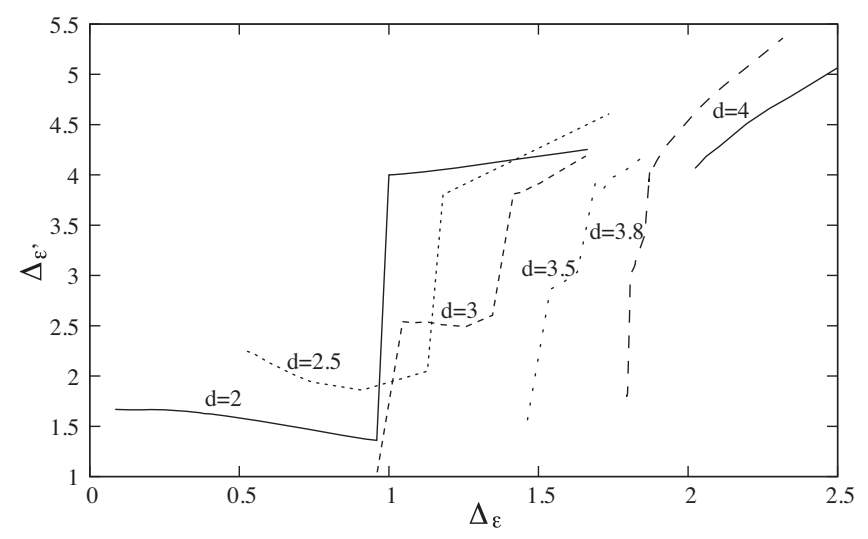

FIG. 6. Plots of the Rychkov bound in various noninteger dimensions consist of two plateaus. The higher plateau is associated with the Ising universality class, while the lower one is associated with the tricritical Ising universality class. The lower plateau is smaller for higher dimensions and vanishes at four dimensions. 

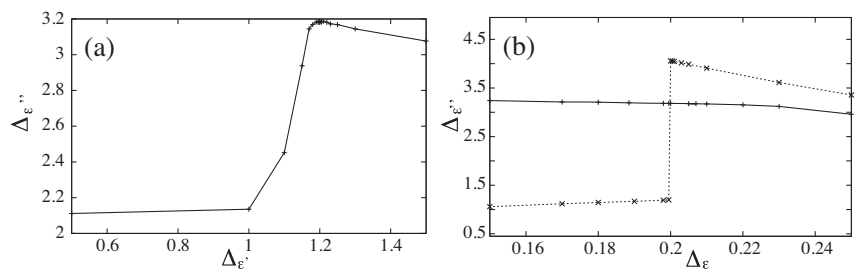

FIG. 7. (a) Upper bound on $\Delta_{\epsilon^{\prime \prime}}$ from the bootstrap constraints associated with the $\langle\sigma \sigma \sigma \sigma\rangle$ correlator in two dimensions for $\Delta_{\sigma}=0.075, \Delta_{\epsilon}=0.2$. (b) Upper bound on $\Delta_{\epsilon^{\prime \prime}}$ from the bootstrap constraints associated with the $\langle\sigma \sigma \sigma \sigma\rangle$ correlator (solid line) and the $\langle\epsilon \epsilon \epsilon \epsilon\rangle$ correlator (dashed line) in two dimensions for $\Delta_{\sigma}=0.075, \Delta_{\epsilon}=0.2$.

on $\Delta_{\epsilon^{\prime \prime}}$ for a given value of $\Delta_{\epsilon^{\prime}}$. This bound on $\Delta_{\epsilon^{\prime \prime}}$ as a function of $\Delta_{\epsilon^{\prime}}$ is plotted in Fig. 7(a), and we observe that the bound on $\Delta_{\epsilon^{\prime \prime}}$ shows a discontinuous jump to $\approx 3.1$ at $\Delta_{\epsilon^{\prime}}=1.2$. Noting that the minimal model values for $\epsilon^{\prime \prime}$ and $\epsilon^{\prime}$ are 3.0 and 1.2 , respectively, this discontinuous jump occurs close to the exact values.

In another computation, we fix $\Delta_{\sigma}$ and $\Delta_{\epsilon^{\prime}}$ to their minimal model values. Constraints from the $\langle\sigma \sigma \sigma \sigma\rangle$ and the $\langle\epsilon \epsilon \epsilon \epsilon\rangle$ correlators give upper bounds on $\Delta_{\epsilon^{\prime \prime}}$ as a function of $\Delta_{\epsilon}$ which are plotted in Fig. 7(b) as the solid and dotted lines, respectively. When one traces the lower of the two upper bounds, one finds a jump at $\Delta_{\epsilon}=0.2$ where $\Delta_{\epsilon^{\prime \prime}}$ is $\approx 3.1$. Again these are very close to the exact minimal model values.

Thus, we show that in two dimensions, the tricritical Ising CFT seems to be characterized by the property that $\epsilon^{\prime \prime}$ becomes irrelevant as a function of $\Delta_{\epsilon^{\prime}}$ while keeping both $\Delta_{\sigma}$ and $\Delta_{\epsilon}$ fixed-much like the Ising CFT [11] which is characterized by the property that $\epsilon^{\prime}$ goes from being relevant to irrelevant, as a function of $\Delta_{\epsilon}$, while keeping $\Delta_{\sigma}$ fixed. Hence, we conclude that the tricritical Ising CFT could also be isolated by using a bootstrap analysis much like the Ising CFT. The analysis so far, involving the $\epsilon^{\prime \prime}$ operator, has been in two dimensions only where exact results are available. Isolating the tricritical Ising CFT completely in dimensions beyond two, using considerations from the $\epsilon^{\prime \prime}$ operator, would constitute progress.

\section{Multicriticality}

In this section, so far we have (mostly) studied the bootstrap constraints from only one four-point function viz. the pure $\langle\sigma \sigma \sigma \sigma\rangle$ correlator, and this involves only the subset of the CFT data that occur in the $\sigma \times \sigma$ OPE. We now show that these constraints could encode even more information, even of higher-order critical points. First, to reiterate, we have seen how the Ising CFT is characterized by the property that $\epsilon^{\prime}$ goes from being relevant to irrelevant, as a function of $\Delta_{\epsilon}$, while keeping $\Delta_{\sigma}$ fixed. We have also shown that the tricritical Ising CFT is characterized by the property that $\epsilon^{\prime \prime}$ becomes irrelevant as a function of $\Delta_{\epsilon^{\prime}}$, keeping both $\Delta_{\sigma}$ and $\Delta_{\epsilon}$ fixed. Next, one introduces the operator viz. $\epsilon^{\prime \prime \prime}$ and studies the class of CFTs with the OPE $\sigma \times \sigma=\mathbf{1}+\epsilon+\epsilon^{\prime}+\epsilon^{\prime \prime}+\epsilon^{\prime \prime \prime}+\cdots$. The next higher-order critical point would be where the $\Delta_{\epsilon^{\prime \prime \prime}}$ goes from relevant to irrelevant as a function of $\Delta_{\epsilon^{\prime \prime}}$, keeping $\Delta_{\sigma}, \Delta_{\epsilon}$, and $\Delta_{\epsilon^{\prime}}$ fixed. We hope to report investigations along these lines in a future work [50]. Other studies of critical and multicritical models using CFT and other methods include [51-53].

\section{CONCLUSION}

In conclusion, we have shown that the tricritical point, which is known to be unstable because the system can easily cross over to the ordinary critical point, can be studied using conformal bootstrap techniques. Such nonperturbative CFT methods are even more significant for the tricritical point, as the success with $\epsilon$-expansion has been limited. Using the bootstrap constraints coming from only two pure correlators on the CFT data contained in two OPEs, we have seen many signatures of tricritical physics and also obtained the precise value of one critical exponent. To get precise values for the other exponents, one will have to consider crossing symmetry constraints coming from all correlators, pure and mixed, which would involve CFT data in other OPEs [50].
[1] A. A. Belavin, A. M. Polyakov, and A. B. Zamolodchikov, Infinite conformal symmetry in two-dimensional quantum field theory, Nucl. Phys. B241, 333 (1984).

[2] P. Di Francesco, P. Mathieu, and D. Senechal, Conformal Field Theory (Springer-Verlag, New York, 1997), https:// inspirehep.net/literature/454643.

[3] F. A. Dolan and H. Osborn, Conformal four point functions and the operator product expansion, Nucl. Phys. B599, 459 (2001).
[4] F. A. Dolan and H. Osborn, Conformal partial waves and the operator product expansion, Nucl. Phys. B678, 491 (2004).

[5] F. A. Dolan and H. Osborn, Conformal partial waves: Further mathematical results, arXiv:1108.6194.

[6] M. Hogervorst and S. Rychkov, Radial coordinates for conformal blocks, Phys. Rev. D 87, 106004 (2013).

[7] F. Kos, D. Poland, and D. Simmons-Duffin, Bootstrapping the $O(N)$ vector models, J. High Energy Phys. 06 (2014) 091. 
[8] F. Kos, D. Poland, and D. Simmons-Duffin, Bootstrapping mixed correlators in the 3D Ising model, J. High Energy Phys. 11 (2014) 109.

[9] D. Poland, S. Rychkov, and A. Vichi, The conformal bootstrap: Theory, numerical techniques, and applications, Rev. Mod. Phys. 91, 015002 (2019).

[10] R. Rattazzi, V.S. Rychkov, E. Tonni, and A. Vichi, Bounding scalar operator dimensions in 4D CFT, J. High Energy Phys. 12 (2008) 031.

[11] S. Rychkov, Conformal bootstrap in three dimensions?, arXiv:1111.2115.

[12] S. El-Showk, M. F. Paulos, D. Poland, S. Rychkov, D. Simmons-Duffin, and A. Vichi, Solving the 3D Ising model with the conformal bootstrap, Phys. Rev. D 86, 025022 (2012).

[13] S. El-Showk, M. F. Paulos, D. Poland, S. Rychkov, D. Simmons-Duffin, and A. Vichi, Solving the 3D Ising model with the conformal bootstrap II. $c$-minimization and precise critical exponents, J. Stat. Phys. 157, 869 (2014).

[14] S. El-Showk, M. Paulos, D. Poland, S. Rychkov, D. Simmons-Duffin, and A. Vichi, Conformal Field Theories in Fractional Dimensions, Phys. Rev. Lett. 112, 141601 (2014).

[15] F. Kos, D. Poland, D. Simmons-Duffin, and A. Vichi, Bootstrapping the $O(N)$ archipelago, J. High Energy Phys. 11 (2015) 106.

[16] F. Kos, D. Poland, D. Simmons-Duffin, and A. Vichi, Precision islands in the Ising and $O(N)$ models, J. High Energy Phys. 08 (2016) 036.

[17] N. Bobev, S. El-Showk, D. Mazac, and M. F. Paulos, Bootstrapping the Three-Dimensional Supersymmetric Ising Model, Phys. Rev. Lett. 115, 051601 (2015).

[18] F. Gliozzi, More Constraining Conformal Bootstrap, Phys. Rev. Lett. 111, 161602 (2013).

[19] Y. Nakayama, Bootstrapping Critical Ising Model on ThreeDimensional Real Projective Space, Phys. Rev. Lett. 116, 141602 (2016).

[20] R. Gopakumar, A. Kaviraj, K. Sen, and A. Sinha, Conformal Bootstrap in Mellin Space, Phys. Rev. Lett. 118, 081601 (2017).

[21] R. B. Griffiths, Thermodynamics Near the Two-Fluid Critical Mixing Point in $\mathrm{He}^{3}-\mathrm{He}^{4}$, Phys. Rev. Lett. 24, 715 (1970).

[22] I. D. Lawrie, Tricritical scaling and renormalisation of $\phi^{6}$ operators in scalar systems near four dimensions, J. Phys. A 12, 919 (1979).

[23] A. L. Lewis and F. W. Adams, Tricritical behavior in two dimensions. 2. Universal quantities from the epsilon expansion, Phys. Rev. B 18, 5099 (1978).

[24] J. S. Hager, Six-loop renormalization group functions of $\mathrm{O}(\mathrm{n})$-symmetric $\Phi^{6}$-theory and epsilon-expansions of tricritical exponents up to $\varepsilon^{3}$, J. Phys. A 35, 2703 (2002).

[25] K. G. Wilson, Non-Lagrangian models of current algebra, Phys. Rev. 179, 1499 (1969).

[26] L. P. Kadanoff, Operator Algebra and the Determination of Critical Indices, Phys. Rev. Lett. 23, 1430 (1969).

[27] I. D. Lawrie and S. Serbach, Theory of Tricritical Points, edited by C. Domb and J. L. Lebowitz (Academic Press, New York, 1984), Vol. 9.

[28] M. F. Paulos, JuliBootS: A hands-on guide to the conformal bootstrap, arXiv:1412.4127.
[29] D. Simmons-Duffin, A semidefinite program solver for the conformal bootstrap, J. High Energy Phys. 06 (2015) 174.

[30] C. Behan, PyCFTBoot: A flexible interface for the conformal bootstrap, Commun. Comput. Phys. 22, 1 (2017).

[31] Y. Nakayama and T. Ohtsuki, Conformal Bootstrap Dashing Hopes of Emergent Symmetry, Phys. Rev. Lett. 117, 131601 (2016).

[32] M. Go and Y. Tachikawa, autoboot: A generator of bootstrap equations with global symmetry, J. High Energy Phys. 06 (2019) 084.

[33] These OPEs are directly inspired by the two-dimensional minimal model OPEs. There can be modifications to the OPEs. For example, in dimensions other than two, the $\epsilon$ operator could be present in the $\epsilon \times \epsilon$ OPE; KramersWannier duality [34], which is the reason for its absence (see Sec. 4.3.1 of [35]), is known to be true only in two dimensions. We thank the referee for this point.

[34] H. A. Kramers and G. H. Wannier, Statistics of the twodimensional ferromagnet. Part 1., Phys. Rev. 60, 252 (1941).

[35] S. Rychkov, EPFL lectures on conformal field theory in $D \geq 3$ dimensions, arXiv:1601.05000.

[36] M. Hogervorst, S. Rychkov, and B. C. van Rees, Truncated conformal space approach in $d$ dimensions: A cheap alternative to lattice field theory?, Phys. Rev. D 91, 025005 (2015).

[37] M. Hogervorst, S. Rychkov, and B. C. van Rees, Unitarity violation at the Wilson-Fisher fixed point in $4-\epsilon$ dimensions, Phys. Rev. D 93, 125025 (2016).

[38] J. Golden and M. F. Paulos, No unitary bootstrap for the fractal Ising model, J. High Energy Phys. 03 (2015) 167.

[39] We thank the referee for this description.

[40] D. Poland, D. Simmons-Duffin, and A. Vichi, Carving out the space of 4D CFTs, J. High Energy Phys. 05 (2012) 110.

[41] K. G. Wilson and M. E. Fisher, Critical Exponents in 3.99 Dimensions, Phys. Rev. Lett. 28, 240 (1972).

[42] K. G. Wilson, Quantum field theory models in less than four-dimensions, Phys. Rev. D 7, 2911 (1973).

[43] J.C. Le Guillou and J. Zinn-Justin, Accurate critical exponents for Ising like systems in non-integer dimensions, in Current Physics-Sources and Comments, edited by J. C. Le Guillou and J. Zinn-Justin (Elsevier, 1990), Vol. 7, pp. 559-564, https://doi.org/10.1016/B978-0-444-88597-5 .50077-6; Accurate critical exponents for Ising like systems in non-integer dimensions, J. Phys. (Les Ulis, Fr.) 48, 19 (1987).

[44] R. Guida and J. Zinn-Justin, Critical exponents of the $N$ vector model, J. Phys. A 31, 8103 (1998).

[45] H. Kleinert, J. Neu, V. Schulte-Frohlinde, K. G. Chetyrkin, and S. A. Larin, Five-loop renormalization group functions of $O(n)$ symmetric $\phi^{4}$ theory and $\epsilon$ expansions of critical exponents up to $\epsilon^{5}$, Phys. Lett. B 272, 39 (1991); Erratum, Phys. Lett. B 319, 545(E) (1993).

[46] J. Zinn-Justin, Quantum field theory and critical phenomena, Int. Ser. Monogr. Phys. 113, 1 (2002).

[47] H. Kleinert and V. Schulte-Frohlinde, Critical Properties of $\phi^{4}$-Theories (World Scientific, River Edge, USA, 2001), p. 489, https://inspirehep.net/literature/578755. 
[48] This is essentially Eq. (23) of Ref. [24], which gives the result for $O(n)$ models, and for the tricritical Ising case that we are interested in, we need to set $n=1$ in that equation.

[49] S. Rychkov and Z. M. Tan, The $\epsilon$-expansion from conformal field theory, J. Phys. A 48, 29FT01 (2015).

[50] C. N. Gowdigere, J. Santara, and Sumedha (to be published).
[51] A. Codello, M. Safari, G. P. Vacca, and O. Zanusso, Leading CFT constraints on multi-critical models in $d>2$, J. High Energy Phys. 04 (2017) 127.

[52] A. Codello, M. Safari, G. P. Vacca, and O. Zanusso, Functional perturbative RG and CFT data in the $\epsilon$-expansion, Eur. Phys. J. C 78, 30 (2018).

[53] A. Codello, M. Safari, G. P. Vacca, and O. Zanusso, New universality class in three dimensions: The critical Blume-Capel model, Phys. Rev. D 96, 081701 (2017). 